\title{
Utilização de exergames e seus efeitos sobre a saúde física de pacientes com diagnóstico de câncer: uma revisão integrativa
}

Use of exergaming and its effects on the physical health of patients diagnosed with cancer: an integrative review

Aplicabilidad de las ecuaciones de referencia brasileñas para la prueba de caminata de 6 minutos en pacientes con cáncer de pulmón

Ana Paula Silva', Elisabete Maria de Oliveira², Rodrigo Okubo ${ }^{3}$, Magnus Benetti ${ }^{4}$

RESUMO | Este estudo teve como objetivo a busca de registros com diferenças significativas a respeito da saúde física de pacientes com câncer ao praticarem exergames. Utilizou-se como método a Revisão Integrativa de Literatura com as palavras chaves "Fisioterapia”, "Realidade Virtual”, "Wii", "Exergames" e "Câncer", em português e inglês, nas bases de dados PubMed, Science Direct, Medline, Scopus, Lilacs, SciELO, PEDro, Cochrane e Periódicos Capes. Os critérios de inclusão foram: estudos que utilizassem exergames em tratamentos clínicos e que apresentassem diferenças sobre a saúde física de pacientes com diagnóstico de câncer, de ambos os sexos, de todas as idades e tipos de câncer, publicados nos últimos 10 anos, em qualquer idioma. Dos 3172 artigos encontrados, apenas nove foram incluídos, sendo que esses avaliaram: desempenho físico, sedentarismo, funcionalidade de membros superiores, força muscular de membros inferiores e fadiga. Concluiu-se que os exergames demostraram ser ferramenta promissora para a saúde física de pacientes com diagnóstico de câncer. Descritores | Literatura de Revisão como Assunto; Realidade Virtua; Saúde; Neoplasias Pulmonares; Fisioterapia.

ABSTRACT | This study aimed to search for records with significant differences regarding the physical health of cancer patients when practicing exergaming. The integrative literature review was used as method with the keywords "Physiotherapy," "Virtual Reality," "Wii," “Exergames” and "Cancer," in Portuguese and English, in the following databases: PubMed, Science Direct,
Medline, Scopus, Lilacs, SciELO, PEDro, Cochrane and Capes Journals. The inclusion criteria were studies that used exergaming in clinical treatments and presented differences on the physical health of patients diagnosed with cancer, of both sexes, of all ages and types of cancer, published in the last 10 years, in any language. Of the 3,172 articles found, only nine were included; they evaluated physical performance, sedentary lifestyle, upper limbs functionality, lower limbs muscle strength and fatigue. The review concluded that exergaming is a promising tool for the physical health of patients diagnosed with cancer.

Keywords | Review Literature as Topic ; Lung Neoplasms; Health; Phisiotherapy.

RESUMEN | El objetivo de este estudio fue verificar el impacto de diferentes ecuaciones de referencia brasileñas para la distancia recorrida en la prueba de caminata de 6 minutos (PC6min) en la evaluación de la capacidad de ejercicio funcional en pacientes con cáncer de pulmón (CP). Este estudio transversal incluyó

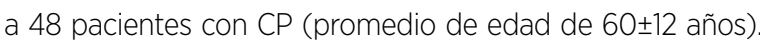
Se evaluó las características sociodemográficas, clínicas y la capacidad funcional de los participantes para ejercitarse en la PC6min según los criterios internacionales. Se analizaron cinco ecuaciones. La distancia recorrida por los pacientes (503 \pm 102 metros) estuvo relativamente cerca de las distancias predichas por las ecuaciones de referencia (82-94\% del predicho), aunque son estadísticamente menores ( $p<0,05$ para todas).

Palabras clave | Literatura de Revisión como Asunto; Neoplasias Pulmonares; Salud; Fisioterapia. 


\section{INTRODUÇÃO}

O tratamento do câncer pode gerar efeitos adversos, e cada tipo de tratamento pode levar a uma sequela diferente como náuseas, danos em órgãos, diminuição da densidade óssea, redução da força muscular e da aptidão física, prejuízo na função cardíaca e função pulmonar diminuída $a^{1-5}$. Por consequência, o tratamento pode interferir negativamente na capacidade física e mental dos pacientes de se envolverem em atividades físicas ${ }^{6}$.

O gasto energético diário reduzido e os baixos níveis de atividade física têm sido descritos como a causa mais importante na redução da capacidade de realização de exercícios físicos e de inserção social em pacientes sobreviventes de leucemia e outras neoplasias 5 . Isso pode ser minimizado ou evitado com a introdução de um programa de exercícios durante e após o tratamento oncológico ${ }^{5}$, tendo em vista a existência de evidências mostrando que o exercício é seguro, viável e benéfico em diferentes fases desta reabilitação ${ }^{7}$ - portanto, maneiras simples, baratas e motivadoras de promover atividade física e exercício tornam-se uma parte cada vez mais importante no tratamento do câncer ${ }^{8}$.

Intervenções por meio de atividade física resultam em efeitos na saúde física e mental de pacientes jovens com câncer e sobreviventes. Os benefícios relatados com mais frequência são: $>50 \%$ para força, fadiga e auto eficácia ${ }^{9}$.

A correlação positiva e linear observada entre as variáveis melhoras do condicionamento físico geral e a redução nos níveis de fadiga dos pacientes sugere que a melhora em diversos parâmetros fisiológicos - e não só de um parâmetro, exclusivamente - exerce uma influência positiva na redução da fadiga em pacientes com câncer ${ }^{10}$.

A combinação dos treinamentos de força e aeróbio pode trazer benefícios para os pacientes em tratamento e póstratamento de câncer, sendo bem tolerada pelos pacientes. Evidentemente, os efeitos positivos do exercício podem variar significativamente para cada indivíduo em função do tipo de câncer, da intensidade da atividade, da frequência e duração do programa de exercícios e do estilo de vida do paciente. São essenciais: a seleção cuidadosa dos pacientes, a supervisão durante o treinamento e o acompanhamento médico ${ }^{3,11}$.

Tem ocorrido, nos últimos anos, uma tendência para o uso de videogames em aplicações de saúde. Os videogames interativos - também conhecidos como exergames possibilitam o indivíduo a interagir com o jogo, movendo seus membros ou todo o corpo. Esse recurso apresenta uma variedade de aplicações possíveis no campo da medicina e da reabilitação ${ }^{12}$ : a natureza muitas vezes aborrecida e repetitiva do exercício de reabilitação pode ser transformada em uma atividade em que os pacientes aderem felizmente por meio do uso de videogames, que promovem maior entretenimento e diversão ao serem jogados ${ }^{12}$.

Dependendo do design do jogo, os exergames podem ser usados para melhorar a aptidão cardiorrespiratória ou o controle sensório-motor ${ }^{13}$. Evidências da efetividade das intervenções com exergames em pessoas com alguma deficiência ainda são limitadas, porém promissoras ${ }^{13}$. Estudos indicam que os exergames têm efeitos positivos tanto na motivação à participação ativa na reabilitação quanto nas funções prejudicadas ${ }^{14}$.

Os videogames são capazes de promover atividade física para indivíduos que necessitam melhorar o equilibrio, que estejam em processo de reabilitação por doenças agudas ou crônicas e que apresentem deficiência física ou atraso no desenvolvimento motor ${ }^{15}$. Além disso, os jogos possibilitam a realização de uma atividade física em pacientes oncológicos que necessitam de cuidados especiais quanto ao risco de infecções, podendo ser reproduzidos no próprio quarto do paciente ${ }^{8,16}$.

Levando em conta o destaque que se tem dado ao fator de risco sedentarismo para a manifestação de quadros de doenças crônicas (e até mesmo o câncer), e considerando que o paciente oncológico vive uma particularidade cotidiana - que é ser submetido a quimioterapia regularmente, passando por períodos de baixa imunidade -, deduz-se que o uso dos exergames, em ambientes seguros e livres de intempéries da natureza, pode se apresentar como solução ideal para a retomada da prática de exercícios físicos regulares ${ }^{17}$.

Argumenta-se que a intervenção pelo programa de exercícios da plataforma digital pode ser viável e traz evidências preliminares de benefícios para o humor e a fadiga em pacientes pediátricos com câncer. Ressalta-se que a eficácia da atividade física em oncologia pediátrica é promissora, mas exige pesquisas continuadas em pacientes cujos comportamento sedentário e efeitos colaterais associados são uma preocupação crescente ${ }^{18}$.

A educação da pessoa com doença oncológica sobre os benefícios do exercício no controle da fadiga deve ser considerada desde o início do processo de tratamento, bem como a sua inclusão em programas de exercícios para os indivíduos com câncer e fadiga associada ${ }^{19}$.

$O$ fato de os pacientes poderem continuar a se exercitar com auxílio do videogame em sua própria casa, mesmo após a alta hospitalar, é um benefício potencial adicional ao uso da tecnologia durante a reabilitação ${ }^{12,20}$; os videogames podem oferecer um meio para fisioterapeutas avaliarem remotamente a aderência dos pacientes em sua terapia e monitorar as mudanças na função ao longo do tempo ${ }^{12}$. 
O objetivo deste estudo foi, portanto, encontrar evidências na literatura que apontassem diferenças significativas sobre a saúde física de pacientes com câncer ao praticar exergames.

\section{METODOLOGIA}

Este estudo trata-se de uma Revisão Integrativa de Literatura (RI), que é um método de revisão específico que sintetiza um assunto para promover maiores entendimento e compreensão de um fenômeno particular ou uma questão relacionada à saúde, permitindo uma ampla análise da literatura ${ }^{21}$.A RI permite a busca, a avaliação crítica e a síntese do tema investigado, podendo tornar os resultados de pesquisas mais acessíveis, reduzindo alguns obstáculos da utilização do conhecimento científico e possibilitando, ao leitor, o acesso a diversos estudos realizados sobre um único tema ${ }^{22,23}$.

As buscas pelos estudos foram realizadas entre os meses maio e julho de 2017. Nove bases de dados foram definidas como fonte de levantamento dos estudos: PubMed, SciELO, Lilacs, MEDLINE, Science Direct, Scopus, PEDro, Cochrane e Periódicos CAPES. As pesquisas foram realizadas utilizando os seguintes descritores (em português e inglês): "Fisioterapia"; "Realidade Virtual"; "Wii”; "Exergames"; "Câncer" e o operador booleano AND.

Incluiu-se, nesta revisão, estudos que contemplaram a utilização de exergames em colaboração com tratamentos clínicos que apresentassem diferença sobre a saúde física de pacientes com diagnóstico de câncer, de ambos os sexos, de todas as idades e tipos de câncer, publicados nos últimos 10 anos, em qualquer idioma.

Foram excluídos desta pesquisa artigos que não estivessem disponibilizados na íntegra, artigos duplicados, artigos que não utilizassem exergames ou que não se enquadrassem nos objetivos do presente estudo.

\section{RESULTADOS}

A pesquisa identificou originalmente 3172 artigos que, após seguidos os passos metodológicos de avaliação, foram reduzidos a nove estudos que atendiam aos critérios de inclusão relatados na metodologia. Os passos metodológicos que levaram a este resultado encontram-se na Figura 1.

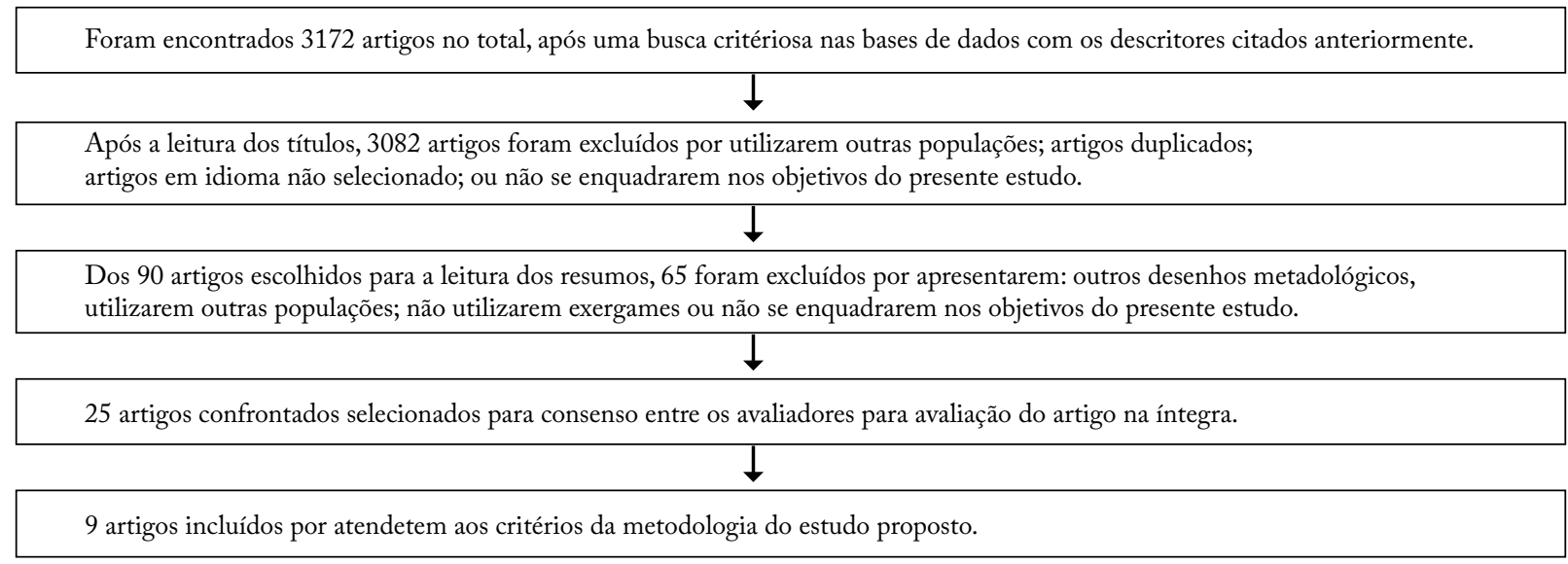

Figura 1. Diagrama com a distribuição dos artigos de acordo com os descritores e os critérios de inclusão e exclusão

Apresentaram repercussões sobre a saúde física de pacientes os estudos que avaliaram: desempenho físico $(\mathrm{n}=3)$, funcionalidade de membros superiores $(\mathrm{n}=2)$, força muscular de membros inferiores $(n=1)$, fadiga $(n=3)$ e sedentarismo $(n=3)$.

Todos os estudos incluídos eram ensaios clínicos. Seis estudos foram realizados em pacientes com idade superior a 18 anos, dois foram conduzidos em jovens menores de 18 anos e um utilizou adolescentes e adultos jovens (13 a 25 anos). Em termos de plataformas de jogos, a maioria dos estudos (n=7) usou o Nintendo Wii ${ }^{\mathrm{TM}}$ (Kyoto, Japão), apenas um estudo usou o Xbox360 Kinect ${ }^{\mathrm{TM}}$ (Microsoft, Redmond, WA), e um utilizou o IREX System.

A frequência de exergaming mínima, presente na maioria dos estudos, foi de 30 minutos por dia, três vezes por semana. Os tipos de câncer inclusos nos estudos foram tumor cerebral $(\mathrm{n}=2)$, neoplasias hematológicas $(\mathrm{n}=1)$, receptores de transplante de células estaminais hematopoiéticas ( $n=1)$, câncer de pulmão de células nãopequenas $(n=1)$, câncer de mama $(n=1)$, além dos artigos que incluíram pacientes com qualquer tipo de câncer $(n=3)$. Os demais resultados encontram-se no Quadro 1. 


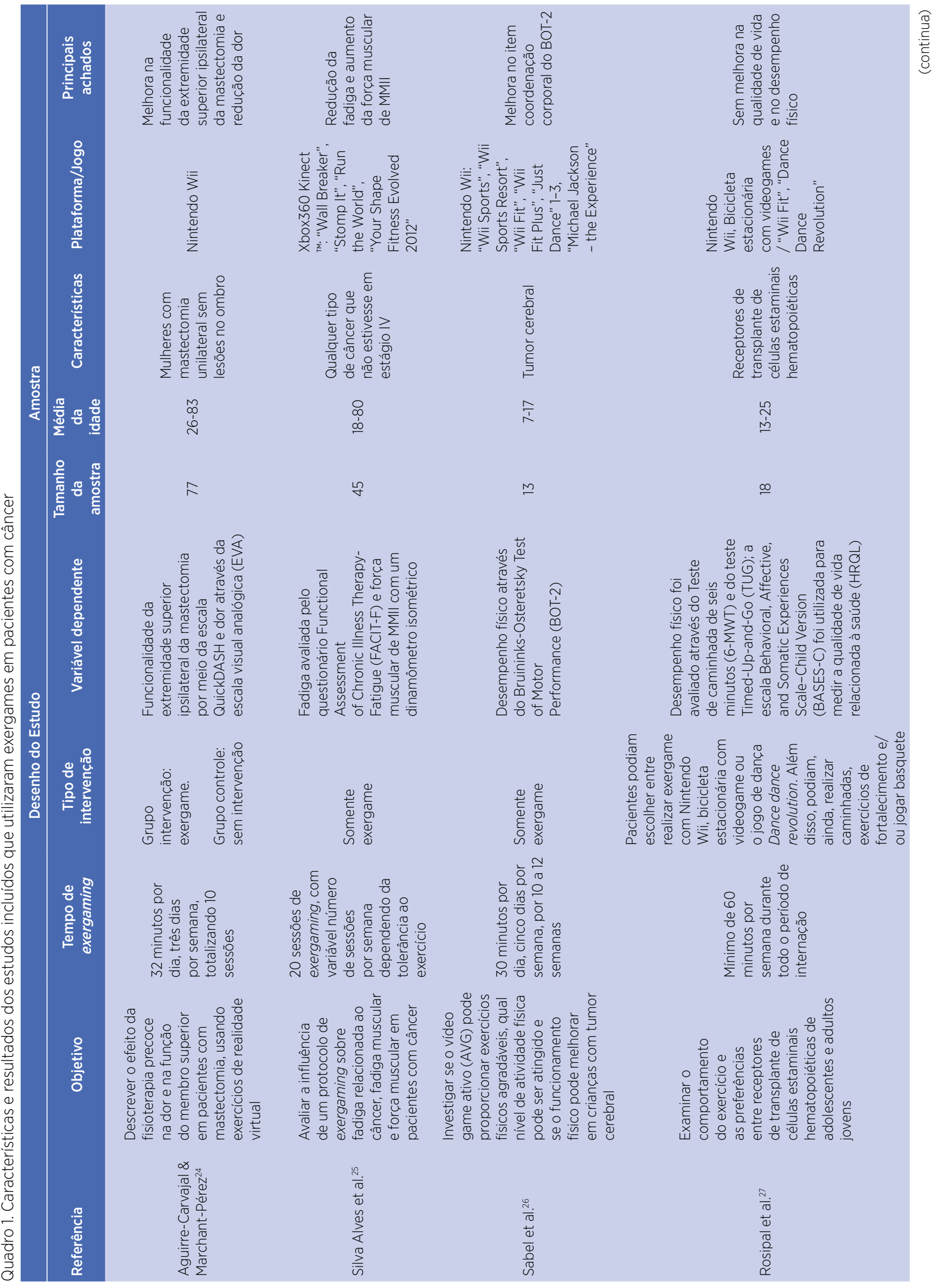




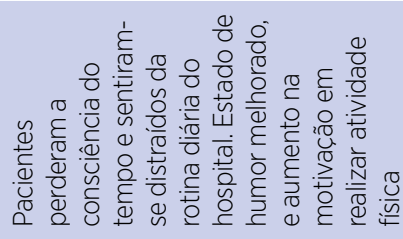

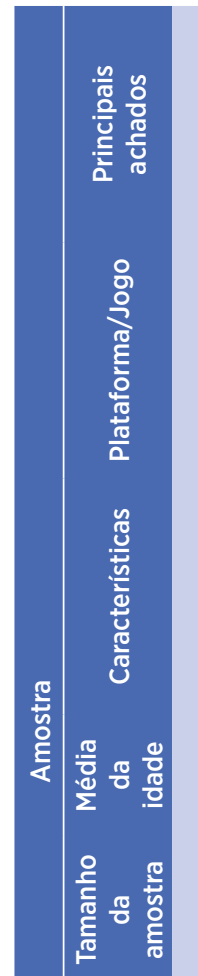

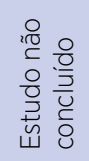
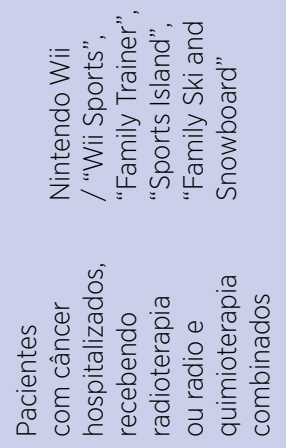

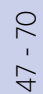



$\frac{0}{\frac{0}{0}}$

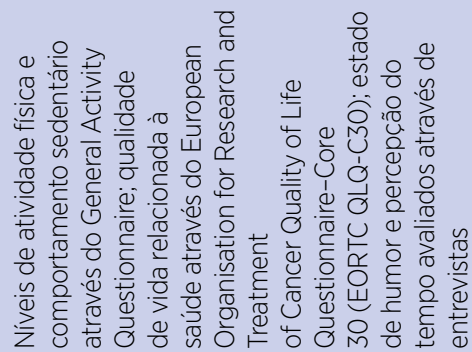

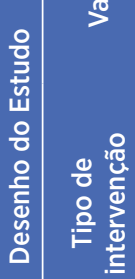

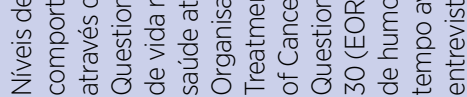

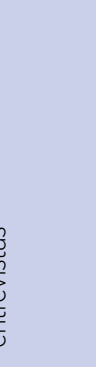

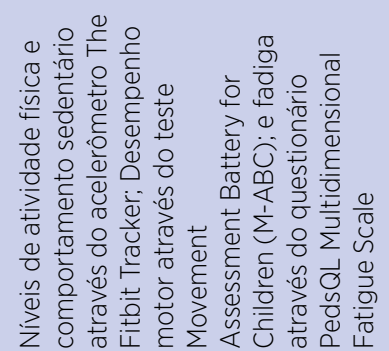

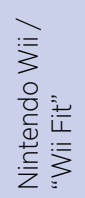

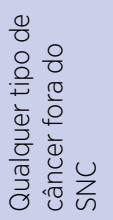

$\frac{6}{m}$

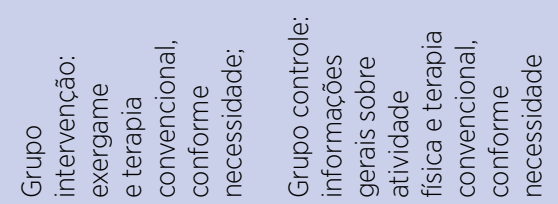

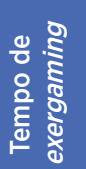

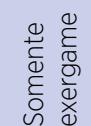

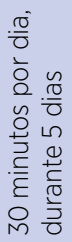

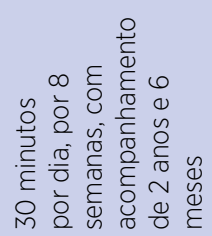

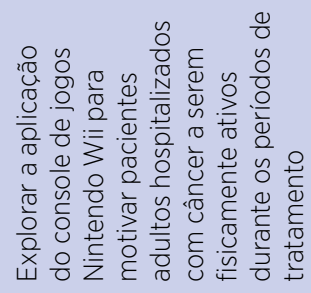
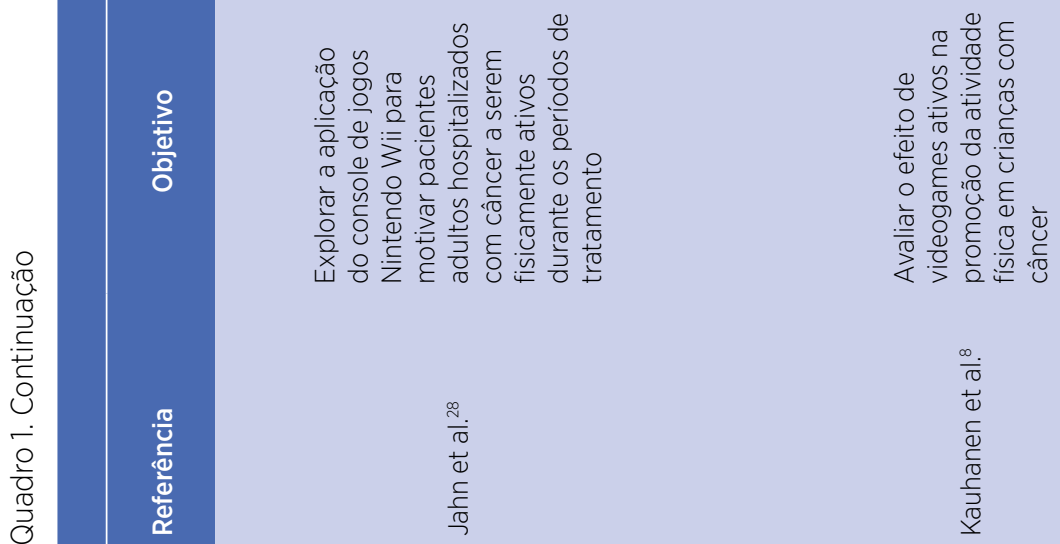
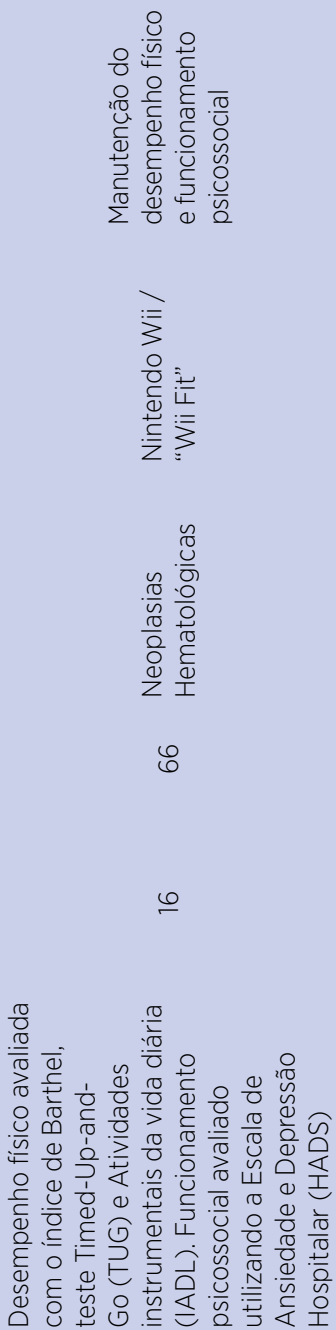

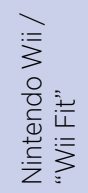

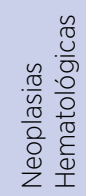

6

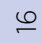

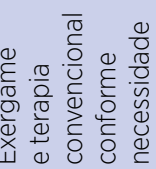

$\frac{\pi}{\frac{\pi}{0}} \underset{\frac{\pi}{0}}{\frac{\pi}{\pi}} \stackrel{0}{\frac{\pi}{\pi}}$

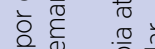

थิ

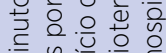

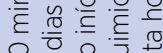

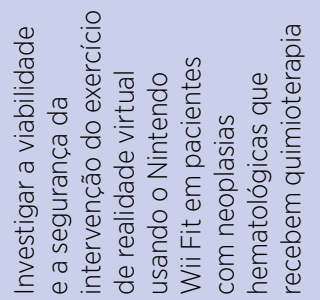




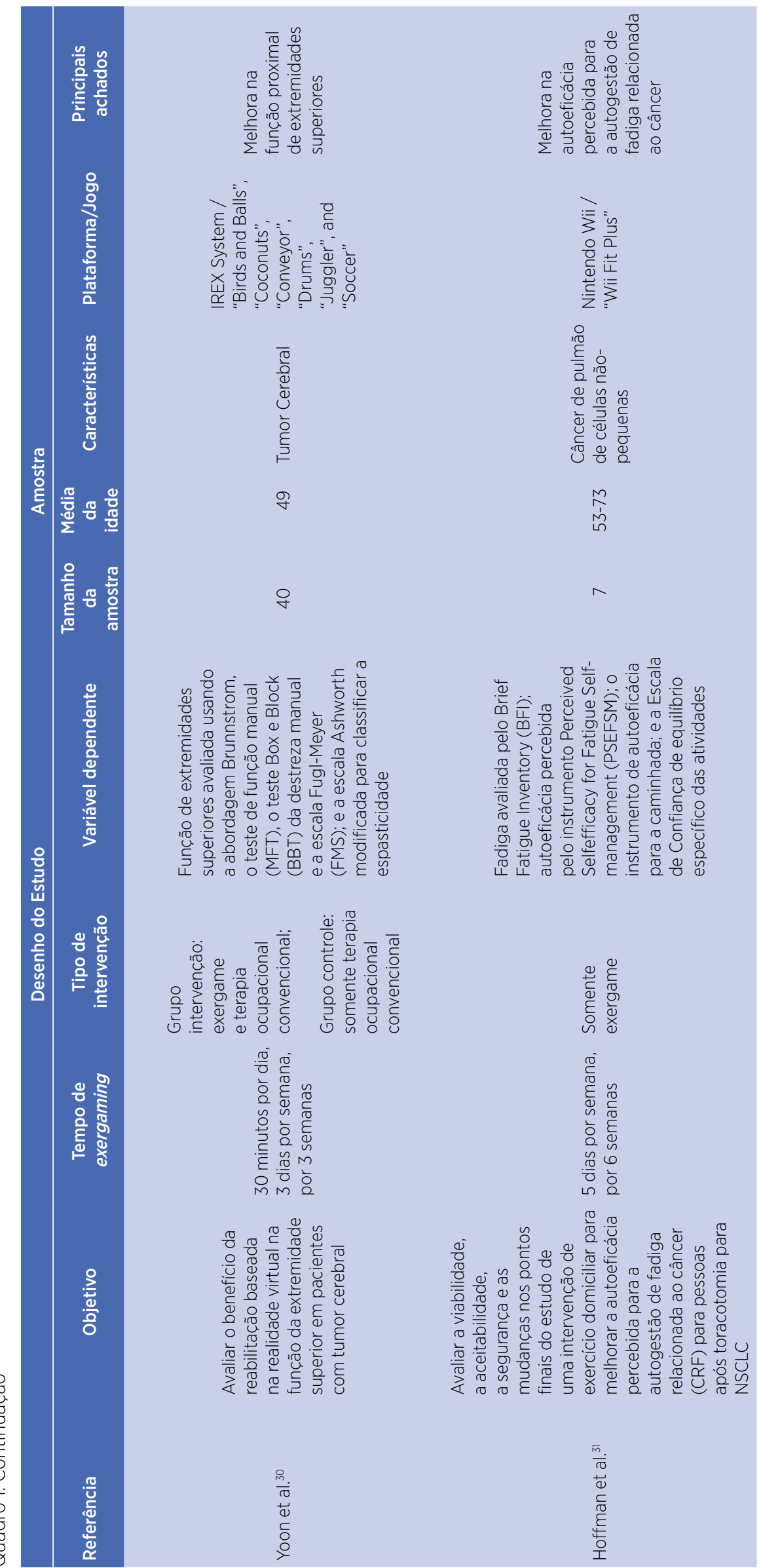




\section{DISCUSSÃO}

De modo geral, a grande maioria dos estudos demonstrou resultados promissores quanto aos efeitos sobre a saúde física relacionados à terapia. Os jogos que se concentraram em habilidades ou déficits específicos revelaram-se particularmente úteis na ajuda aos pacientes para alcançar os objetivos da reabilitação ${ }^{24-27}$.

Os exergames utilizados nos estudos mostraram-se aceitáveis e desfrutáveis por quase todas as populações incluídas nesta revisão, incluindo populações idosas com pouca ou nenhuma exposição prévia aos jogos de videogame. Nenhum evento adverso grave foi relatado nos estudos analisados. A natureza divertida e distrativa dos exergames pode ser útil para complementar a terapia e restaurar o humor, além das melhorias físicas reais alcançadas.

\section{Desempenho físico}

Quanto aos efeitos sobre a saúde física, cada um dos estudos abordou uma variável diferente. $\mathrm{O}$ estudo de Sabel et al. ${ }^{26}$, membros do Departamento de Pediatria no Institute of Clinical Sciences, avaliou o desempenho físico através do Bruininks-Osteretsky Test of Motor Performance, segunda edição (BOT-2), e encontrou diferença significativa nos valores de coordenação corporal de antes e após a intervenção com exergame em crianças com tumores cerebrais.

Por outro lado, Tsuda et al..$^{28}$, da divisão de Hematologia e Reumatologia da Universidade Teikyo Chiba Medical Center, no Japão, verificaram que houve uma manutenção do desempenho físico em pacientes com neoplasias hematológicas, em que o índice de Barthel, a força do punho, força de extensão do joelho, tempo de permanência em uma perna, pontuação do TUG e o questionário de atividades instrumentais da vida diária obtiveram valores sem mudanças significativas antes e após a intervenção.Já Rosipal et al. ${ }^{29}$, do Texas Children's Hospital, nos Estados Unidos, avaliaram o desempenho físico de pacientes receptores de transplante de células estaminais hematopoiéticas através do Teste de Caminhada de 6 minutos (6-MWT) e do teste Timed-Up-and-Go (TUG), e não obtiveram resultados positivos ao final do estudo.

Em uma população diferente, o estudo realizado por Taylor et al..$^{30}$, da Universidade de Essex, em Colchester, Reino Unido, avaliou o desempenho físico através do teste TUG e do Teste de Caminhada de 10 Minutos, e, após uma intervenção utilizando exergame, observou melhora significativa em idosos com histórico de quedas.

\section{Funcionalidade}

Aguirre-Carvajal e Marchant-Pérez ${ }^{24}$ e Yoon et al. ${ }^{27}$ observaram melhorias significativas pós-tratamento no grau de funcionalidade das extremidades superiores. Enquanto Yoon et al. ${ }^{27}$, do Departamento de Medicina de Reabilitação da Faculdade de Medicina da Universidade de Ulsan, em Seul, utilizaram o teste Box e Block (teste de função manual, escala Fugl-Meyer e a versão coreana do Índice de Barthel Modificado) para avaliar a funcionalidade em pacientes com tumor cerebral, AguirreCarvajal e Marchant-Pérez ${ }^{24}$, da Pontificia Universidad Católica de Valparaíso, no Chile, avaliaram o grau de funcionalidade da extremidade superior ipsilateral de mulheres mastectomizadas através do teste QuickDASH.

Joo et al. ${ }^{31}$, membros do Centro de Reabilitação Tan Tock Seng, em Singapura, utilizaram um programa de exergame em pacientes com sequelas de AVE, em que a função de membros superiores foi avaliada através do FuglMeyer Assessment of Upper Limb Motor Function (FMA), observando melhora significativa após a intervenção.

\section{Fadiga}

Os estudos de Kauhannen et al. ${ }^{8}$, Hoffman et al..$^{32} \mathrm{e} \mathrm{da}$ Silva Alves et al..$^{25}$ observaram os efeitos dos exergames sobre a fadiga. Kauhannen et al. ${ }^{8}$, associada ao Departamento de Ciências da Enfermagem da Universidade de Turku, na Finlândia, utilizaram questionários padronizados da Escala de Fadiga Multidimensional PedsQL para avaliar a fadiga de crianças com câncer (apesar de não ter publicado seus resultados). Hoffman et al..$^{32}$, da Faculdade de Enfermagem da Michigan State University, nos Estados Unidos, avaliaram a fadiga de pacientes com câncer de pulmão de células não-pequenas por meio dos questionários Brief Fatigue Inventory (BFI) e Perceived Selfefficacy for Fatigue Self-management (PSEFSM), obtendo resultados positivos ao final da intervenção.

Já da Silva Alves et al. ${ }^{25}$, doutorandos da Universidade Federal de Alfagas, no Brasil, avaliaram a fadiga relacionada ao câncer usando o questionário Functional Assessment of Chronic Illness Therapy (FACIT-F) e observaram diferença significativa após a intervenção. Em pacientes afro-americanas com lúpus, o estudo de Yuen et al. ${ }^{33}$, do Departamento de Terapia Ocupacional da Universidade do Alabama em Birmingham, avaliaram a percepção de fadiga através do Fatigue Severity Scale (FSS) e, após intervenção utilizando exergames, observou uma redução significativa na fadiga nessa população. 


\section{Sedentarismo}

O sedentarismo é um dos fatores de risco derivados do câncer e, por trazer diversas alterações sobre a saúde física como consequência, foi incluído neste estudo. Desta forma, três artigos avaliaram o comportamento sedentário e os níveis de atividade física de seus pacientes.

Kauhannen et al. ${ }^{8}$ utilizaram o acelerômetro The Fitbit Tracker em crianças com câncer para avaliar os níveis de atividade física e comportamento sedentário, apesar de não ter publicado seus resultados. Jahn et al. ${ }^{34}$, do Hospital Universitário Halle, Universidade Martin Luther HalleWittenberg na Alemanha, usaram o General Activity Questionnaire para avaliar os mesmos parâmetros em pacientes com câncer hospitalizados, e observaram aumento na motivação da realização de atividade física. Sabel et al. ${ }^{26}$, utilizaram o monitor de atividade multisensorial SenseWear Pro 2 armband para registrar os níveis de atividade física de crianças com câncer, e não observaram mudanças nos níveis de sedentarismo comparados aos do início do estudo.

O repouso absoluto aos pacientes com câncer, devido à convicção de que qualquer tipo de atividade física poderia agravar o quadro clínico já deteriorado pelo tratamento ou pela doença, era recomendado em épocas passadas; entretanto, esse pensamento tem sido questionado nos últimos anos, dado que as evidências científicas, ainda que escassas, demonstram que o repouso excessivo, com falta de atividade física, resulta em descondicionamento físico grave, fadiga, redução do estado funcional e do bem-estar dos indivíduos ${ }^{35}$.

A prática de atividade física pode melhorar o desempenho físico, emocional e, consequentemente, a qualidade de vida de pacientes com câncer, tanto durante quanto após o tratamento convencional (quimioterapia e radioterapia) e transplante de medula óssea. Por isso, a atividade física pode ser adicionada como terapia complementar para o tratamento de pacientes com neoplasias malignas como forma de reduzir os níveis de sedentarismo e, assim, evitar as consequências provindas desse comportamento ${ }^{36}$. Tendo em vista os benefícios da atividade física, os exergames surgem como uma alternativa motivacional na promoção de exercícios e do combate ao sedentarismo, que pode ser supervisionado em casa e, para aqueles com baixa autoconfiança, fornece um meio para praticar a atividade física em um ambiente familiar ${ }^{15,37}$.

\section{Força muscular}

Quanto à força muscular, o único estudo que avaliou o parâmetro foi o de Silva Alves et al. ${ }^{27}$, que utilizaram um dinamômetro isométrico nos tornozelos de pacientes com câncer, apresentando resultados positivos na força muscular de membros inferiores ao final da intervenção com exergame.

No estudo de Gschwind et al. ${ }^{38}$, do grupo Assistive Healthcare Information Technology, no Instituto Austríaco de Tecnologia, em Viena, na Áustria, a força muscular foi avaliada através de um dos tópicos do Physiological Profile Assessment (PPA), pelo qual também foi observado o aumento na força muscular de membros inferiores em pessoas idosas após intervenção utilizando exergames. Teodoro ${ }^{39}$ utilizou um protocolo com exergame em um paciente com encefalopatia crônica não progressiva na infância (CNPI) e observou melhora do equilíbrio estático na postura em pé, da força muscular em MMII e coordenação de MSE.

\section{CONCLUSÃO}

Os exergames mostraram-se ferramentas promissoras para a melhoria da saúde física entre pacientes com diagnóstico de câncer. Embora os resultados dos estudos sejam, em sua maioria, positivos quanto aos efeitos físicos, faz-se necessário a realização de um maior número de ensaios clínicos, com maior rigor metodológico, para avaliar se os exergames são efetivos para fins terapêuticos em pacientes com câncer. Vale ressaltar que os avanços nos estudos para determinar a melhor forma de incorporar o exergame em programas convencionais de reabilitação e determinar o protocolo ideal para a duração e frequência de execução dos exergames para a população usuária são de suma importância. O potencial dos exergames para motivar a reabilitação de maneira divertida entre pacientes com diagnóstico de câncer deve ser aproveitado tanto por pesquisadores quanto por fisioterapeutas e demais profissionais da saúde.

\section{REFERÊNCIAS}

1. Oeffinger KC, Mertens AC, Sklar CA, Kawashima T, Hudson MM, Meadows AT, et al. Chronic health conditions in adult survivors of childhood cancer. N Eng J Med. 2006;355(15):1572-82. doi:10.1056/NEJMsa060185

2. Gocha Marchese V, Chiarello LA, Lange BJ. Strength and functional mobility in children with acute Iymphoblastic leukemia. Med Pediatr Oncol. 2003;40(4):230-2. doi:10.1002/ mpo.10266

3. Hartman A, Van den Bos C, Stijnen T, Pieters R. Decrease in peripheral muscle strength and ankle dorsiflexion as long-term 
side effects of treatment for childhood cancer. Pediatr Blood Cancer. 2008;50(4):833-7. doi: 10.1002/pbc.21325

4. Ramchandren S, Leonard M, Mody RJ, Donohue JE, Moyer J, Hutchinson R, et al. Peripheral neuropathy in survivors of childhood acute lymphoblastic leukemia. J Peripher Nerv Syst. 2009;14(3):184-9. doi: 10.1111/j.1529-8027.2009.00230.x

5. Braam KI, Van der Torre P, Takken T, Veening MA, Van Dulmenden Broeder E, Kaspers GJL. Physical exercise training interventions for children and young adults during and after treatment for childhood cancer. Cochrane Database Syst Rev. 2013;(4):CD008796. doi: 10.1002/14651858.cd008796.pub2

6. Braam KI, Van Dijk-Lokkart EM, Kaspers GJL, Takken T, Huisman J, Bierings MB, et al. Cardiorespiratory fitness and physical activity in children with cancer. Support Care Cancer. 2016;24(5):2259-68. doi: 10.1007/s00520-015-2993-1

7. Chamorro-Viña C, Guilcher GM, Khan FM, Mazil K, Schulte F, Wurz A, et al. Exercise in pediatric autologous stem cell transplant patients: a randomized controlled trial protocol. BMC câncer. 2012;12:401. doi: 10.1186/1471-2407-12-401

8. Kauhanen L, Järvelä L, Lähteenmäki PM, Arola M, Heinonen OJ, Axelin A, et al. Active video games to promote physical activity in children with cancer: a randomized clinical trial with follow-up. BMC Pediatr. 2014;14:94. doi: 10.1186/1471-2431-14-94

9. Anzeneder S, Benzing VJ, Pompei L, Schmidt M. Physical and mental health outcomes of physical exercise training in young cancer inpatients and survivors-a systematic review of RCTs. 2020. In: Kind, Sport und Gesundheit. 12. SGS-Jahrestagung; 2020 Feb 6-7. Basel: Die Sportwissenschaftliche Gesellschaft der Schweiz SGS; 2020. doi: 10.7892/boris.144444

10. Battaglini CL, Bottaro M, Campbell JS, Novaes J, Simão R. Atividade física e níveis de fadiga em pacientes portadores de câncer. Rev Bras Med Esporte. 2004;10(2),98-104. doi: 10.1590/ S1517-86922004000200004

11. Nascimento EBD, Leite RD, Prestes J. Câncer: benefícios do treinamento de força e aeróbio. Rev Educ Fis. 2011;22(4),652-8. doi: 10.4025/reveducfis.v22i4.11670

12. Smith ST, Talaei-Khoei A, Ray M, Ray P. Agent-based monitoring of functional rehabilitation using video games. In: Brahnam S, Jain LC, editors. Advanced Computational Intelligence Paradigms in Healthcare 5: intelligent Decision Support Systems. Berlin: Springer; 2010. p. 113-41.

13. Wiemeyer J, Deutsch J, Malone LA, Rowland JL, Swartz MC, Xiong $\mathrm{J}$, et al. Recommendations for the optimal design of exergame interventions for persons with disabilities: challenges, best practices, and future research. Games Health J. 2015;4(1):58-62. doi: 10.1089/g4h.2014.0078

14. Lohse KR, Hilderman CG, Cheung KL, Tatla S, Van der Loos HM. Virtual reality therapy for adults post-stroke: a systematic review and meta-analysis exploring virtual environments and commercial games in therapy. PLoS One. 2014;9(3):e93318. doi: 10.1371/journal.pone.0093318

15. Staiano AE, Flynn R. Therapeutic uses of active videogames: a systematic review. Games Health J. 2014;3(6):351-65. doi: 10.1089/g4h.2013.0100

16. Andrade BA. Assistência fisioterapêutica ao paciente oncológico pediátrico. In: Nicolau CM, Andrade LB, organizadores. Profisio: Programa de Atualização em Fisioterapia Pediátrica e Neonatal: Cardiorrespiratória e Terapia Intensiva: Ciclo 2. Porto Alegre: Artmed; 2013. p. 99-123.
17. Oliveira EM. Avaliação da utilização de exergame direcionado à promoção da saúde de crianças e adolescentes com diagnóstico de câncer [dissertation]. Florianópolis: Universidade do Estado de Santa Catarina; 2020.

18. Platschek AM, Kehe L, Abeln V, Berthold F, Simon T, Struder HK. Computer-based exercise program: effects of a 12-week intervention on mood and fatigue in pediatric patients with cancer. Clin J Oncol Nurs. 2017;21(6):E280-6. doi: 10.1188/17. CJON.E280-E286

19. Rodrigues ACD, Gomes B, Albuquerque C. A intervenção do enfermeiro na implementação de programas baseados no exercício para o controlo da fadiga associada à doença oncológica: uma scoping review. Anais do Simpósio do Exercício e Cancro (OncoFit); 2020, Sep 13-14; Centro de Reabilitação do Norte, Vila Nova de Gaia, Portugal. Ribeira de Pena (Portugal): Desafio Singular; 2020. p. 128. doi: 10.6063/motricidade.20120

20. Chirico A, Lucidi F, De Laurentiis M, Milanese C, Napoli A, Giordano A. Virtual reality in health system: beyond entertainment. $A$ mini-review on the efficacy of VR during cancer treatment. J Cell Physiol. 2016;231(2):275-87. doi: 10.1002/jcp.25117

21. Broome ME. Integrative literature reviews for the development of concepts. In: Rodgers BL, Knafl KA, editores. Concept development in nursing: foundations, techniques and applications. 2nd ed. Philadelphia: Saunders; 1999. p. 231-50.

22. Whittemore $\mathrm{R}$, Knafl $\mathrm{K}$. The integrative review: updated methodology. J Adv Nurs. 2005;52(5):546-53. doi: 10.1111/j.1365-2648.2005.03621.x

23. Mendes KDS, Silveira RCCP, Galvão CM. Revisão integrativa: método de pesquisa para a incorporação de evidências na saúde e na enfermagem. Texto Contexto Enferm. 2008;17(4):758-64. doi: 10.1590/s0104-07072008000400018

24. Aguirre-Carvajal M, Marchant-Pérez P. Descripción del efecto de los ejercicios de la extremidad superior ipsilateral realizados con realidad virtual en mujeres sometidas a mastectomía. Gac Mex Oncol. 2015;14(4):204-9. doi: 10.1016/j.gamo.2015.10.002

25. Silva Alves R, lunes DH, Pereira IC, Borges JBC, Nogueira DA, Silva $A M$, et al. Influence of exergaming on the perception of cancer-related fatigue. Games Health J. 2017;6(2):119-26. doi: 10.1089/g4h.2016.0051

26. Sabel M, Sjölund A, Broeren J, Arvidsson D, Saury JM, Blomgren $\mathrm{K}$, et al. Active video gaming improves body coordination in survivors of childhood brain tumours. Disabil Rehabil. 2016:38(21):2073-84. doi: 10.3109/09638288.2015.1116619

27. Yoon J, Chun MH, Lee SJ, Kim BR. Effect of virtual realitybased rehabilitation on upper-extremity function in patients with brain tumor: controlled trial. Am J Phys Med Rehabil. 2015;94(6):449-59. doi: 10.1097/phm.0000000000000192

28. Tsuda K, Sudo K, Goto G, Takai M, Itokawa T, Isshiki T, et al. A feasibility study of virtual reality exercise in elderly patients with hematologic malignancies receiving chemotherapy. Intern Med. 2016;55(4):347-52. doi: 10.2169/internalmedicine.55.5275

29. Rosipal NC, Mingle L, Smith J, Morris GS. Assessment of voluntary exercise behavior and active video gaming among adolescent and young adult patients during hematopoietic stem cell transplantation. J Pediatr Oncol Nurs. 2013;30(1):24-33. doi: 10.1177/1043454212461071

30. Taylor MJ, Shawis T, Impson R, Ewins K, McCormick D, Griffin M. Nintendo Wii as a training tool in falls prevention 
rehabilitation: case studies. J Am Geriatr Soc. 2012;60(9):1781-3. doi: 10.1111/j.1532-5415.2012.04122.x

31. Joo LY, Yin TS, Xu D, Thia E, Chia PF, Kuah CWK, et al. A feasibility study using interactive commercial off-the-shelf computer gaming in upper limb rehabilitation in patients after stroke. J Rehabil Med. 2010;42(5):437-41. doi: 10.2340/16501977-0528

32. Hoffman AJ, Brintnall RA, Brown JK, Von Eye A, Jones LW, Alderink $\mathrm{G}$, et al. Too sick not to exercise: using a 6-week, home-based exercise intervention for cancer-related fatigue self-management for postsurgical non-small cell lung cancer patients. Cancer Nurs. 2013;36(3):175-88. doi: 10.1097/ncc.0b013e31826c7763

33. Yuen HK, Holthaus K, Kamen DL, Sword DO, Breland HL. Using Wii Fit to reduce fatigue among African American women with systemic lupus erythematosus: a pilot study. Lupus. 2011;20(12):1293-9. doi: 10.1177/0961203311412098

34. Jahn P, Lakowa N, Landenberger M, Vordermark D, Stoll O. InterACTIV: an exploratory study of the use of a game console to promote physical activation of hospitalized adult patients with cancer. Oncol Nurs Forum. 2012;39(2):E84-90. doi: 10.1188/12.onf.e84-e90
35. Cipolat S, Pereira BB, Ferreira FV. Fisioterapia em pacientes com leucemia: revisão sistemática. Rev Bras Cancerol. 2011;2(57):229-36.

36. Dimeo F. Exercise for cancer patients: a new challenge in sports medicine. Brit J Sports Med. 2000;34(3):160-1. doi: 10.1136/ bjsm.34.3.160

37. Taylor MJ, McCormick D, Shawis T, Impson R, Griffin M. Activity-promoting gaming systems in exercise and rehabilitation. J Rehabil Res Dev. 2011;48(10):1171-86. doi: 10.1682/jrrd.2010.09.0171

38. Gschwind YJ, Schoene D, Lord SR, Ejupi A, Valenzuela T, Aal K, et al. The effect of sensor-based exercise at home on functional performance associated with fall risk in older people: a comparison of two exergame interventions. Eur Rev Aging Phys Act. 2015;12:11. doi: 10.1186/s11556-015-0156-5

39. Teodoro FA. Efeito da Wii-reabilitação na função motora, habilidade e independência funcional de escolar com encefalopatia crônica não progressiva da infância: relato de caso [monograph]. Bragança Paulista: Universidade São Francisco; 2011. 